\title{
Long-term survival after intensive chemotherapy or hypomethylating agents in AML patients aged 70 years and older: a large patient data set study from European registries
}

\author{
Christian Récher $\mathbb{D}^{1,19 凶}$, Christoph Röllig $\mathbb{D}^{2,19}$, Emilie Bérard ${ }^{3}$, Sarah Bertoli $\mathbb{D}^{1}{ }^{1}$, Pierre-Yves Dumas $\mathbb{D}^{4}$, Suzanne Tavitian ${ }^{1}$, \\ Michael Kramer ${ }^{2}$, Hubert Serve ${ }^{5}$, Martin Bornhäuser ${ }^{2}$, Uwe Platzbecker (D) ${ }^{6}$, Carsten Müller-Tidow (iD) ${ }^{7}$, Claudia D. Baldus $^{8}$, \\ David Martínez-Cuadrón (DD ${ }^{9}$, Josefina Serrano ${ }^{10}$, Pilar Martínez-Sánchez ${ }^{11}$, Eduardo Rodríguez Arbolí ${ }^{12}$, Cristina Gil ${ }^{13}$, Juan Bergua ${ }^{14}$, \\ Teresa Bernal ${ }^{15}$, Adolfo de la Fuente Burguera ${ }^{16}$, Eric Delabesse (iD ${ }^{17}$, Audrey Bidet ${ }^{18}$, Arnaud Pigneux ${ }^{4,19}$ and Pau Montesinos (D) $^{9,19}$
}

(c) The Author(s) 2021

The outcome of acute myeloid leukemia patients aged 70 years or older is poor. Defining the best treatment option remains controversial especially when choosing between intensive chemotherapy and hypomethylating agents. We set up a multicentric European database collecting data of 3700 newly diagnosed acute myeloid leukemia patients $\geq 70$ years. The primary objective was to compare overall survival in patients selected for intensive chemotherapy $(n=1199)$ or hypomethylating agents $(n=1073)$. With a median follow-up of 49.5 months, the median overall survival was 10.9 (95\% Cl: $9.7-11.6)$ and 9.2 months (95\% Cl: 8.3-10.2) with chemotherapy and hypomethylating agents, respectively. Complete remission or complete remission with incomplete hematologic recovery was $56.1 \%$ and $19.7 \%$ with chemotherapy and hypomethylating agents, respectively $(P<0.0001)$. Treatment effect on overall survival was time-dependent. The Royston and Parmar model showed that patients treated with hypomethylating agents had a significantly lower risk of death before 1.5 months of follow-up; no significant difference between 1.5 and 4.0 months, whereas patients treated with intensive chemotherapy had a significantly better overall survival from four months after start of therapy. This study shows that intensive chemotherapy remains a valuable option associated with a better long-term survival in older AML patients.

Leukemia (2022) 36:913-922; https://doi.org/10.1038/s41375-021-01425-9

\section{INTRODUCTION}

The prognosis of acute myeloid leukemia (AML) patients aged $\geq 70$ years is particularly poor. In the United States, five-year overall survival is $7.4 \%$ and $3.3 \%$ in patients aged $70-74$ and $75-79$, respectively [1]. Similar results have been reported in Europe [2]. Indeed, these patients accumulate adverse risk factors related both to aging and to distinct disease characteristics [3]. Older patients more often have co-morbidities and/or a more compromised performance status at diagnosis [4, 5]. In addition, they more often present disease-related characteristics associated with a poorer prognosis such as complex karyotype, $A S X L 1, R U N X 1$ or TP53 mutations and/or secondary AML evolving from myelodysplastic syndrome or myeloproliferative disorders or after previous exposure to cytotoxic treatments (therapy-related AML) $[6,7]$. This results in poor treatment tolerance, higher treatment-related mortality and more failure or relapse than in younger subjects and explains why recent improvement in overall survival observed in $\mathrm{AML}$ is less pronounced in older patients $[1,8,9]$.

In elderly patients, intensive chemotherapy (IC), hypomethylating agents (HMAs), low-dose cytarabine (LDAC) or best supportive

\footnotetext{
${ }^{1}$ Centre Hospitalier Universitaire de Toulouse, Institut Universitaire du Cancer de Toulouse Oncopole, Université Toulouse III Paul Sabatier, Toulouse, France. ${ }^{2}$ Medizinische Klinik und Poliklinik I, Universitätsklinikum TU Dresden, Dresden, Germany. ${ }^{3}$ Centre Hospitalier Universitaire de Toulouse, Service d'Epidémiologie, CERPOP, Inserm, Université Toulouse III Paul Sabatier, Toulouse, France. ${ }^{4} \mathrm{CHU}$ Bordeaux, Service d'Hématologie Clinique et de Thérapie Cellulaire, Université de Bordeaux, Institut National de la Santé et de la Recherche Médicale, U1035, 33000 Bordeaux, France. ${ }^{5}$ Medizinische Klinik 2, Universitätsklinikum Frankfurt, Frankfurt/Main, Germany. ${ }^{6}$ Klinik und Poliklinik für Hämatologie, Zelltherapie und Hämostaseologie, Universitätsklinikum Leipzig, Leipzig, Germany. ${ }^{7}$ Klinik für Hämatologie, Onkologie und Rheumatologie, Universitätsklinikum Heidelberg, Heidelberg, Germany. ${ }^{8}$ Klinik für Innere Medizin II, Universitätsklinikum Schleswig-Holstein, Kiel, Germany. ${ }^{9}$ Instituto de Investigación Sanitaria La Fe (IISLAFE), Hospital Universitari i Politècnic La Fe, Valencia, Spain. ${ }^{10}$ Hospital Universitario Reina Sofía-IMIBIC, Córdoba, Spain. ${ }^{11}$ Hospital Universitario 12 de Octubre, Madrid, Spain. ${ }^{12}$ Hospital Universitario Virgen del Rocío, Sevilla, Spain. ${ }^{13} \mathrm{Hospital}$ General Universitario de Alicante, Alicante, Spain. ${ }^{14}$ Hospital San Pedro Alcántara, Cáceres, Spain. ${ }^{15}$ Hospital Universitario Central de Asturias, Asturias, Spain. ${ }^{16} \mathrm{MD}$ Anderson Cancer Center Madrid, Madrid, Spain. ${ }^{17}$ Centre Hospitalier Universitaire de Toulouse, Institut Universitaire du Cancer de Toulouse Oncopole, Laboratoire d'Hématologie Biologique, Toulouse, France. ${ }^{18} \mathrm{CHU}$ Bordeaux, Laboratoire d'Hématologie Biologique, F-33000 Bordeaux, France. ${ }^{19}$ These authors contributed equally: Christian Récher, Christoph Röllig, Arnaud Pigneux, Pau Montesinos. ${ }^{凶}$ email: recher.christian@iuct-oncopole.fr
} 
care (BSC) represent standard treatment options. More recently, the addition of the $\mathrm{Bcl} 2$ inhibitor venetoclax to azacitidine or lowdose cytarabine has shown efficacy by improving response to treatment and overall survival in patients judged ineligible for intensive chemotherapy $[10,11]$.

Accurately determining which kind of treatment is most appropriate for which patient remains a daily challenge, particularly in selecting older patients who are suitable for intensive treatment, which is the only therapeutic option associated with long term survival [12]. Scoring systems have been proposed to rationalize clinical decision-making [4, 13-19]. However, many patients and physicians are reluctant to use induction chemotherapy due to its toxicity and disappointing results, especially in patients over 75 years of age [20]. In fact, age $\geq 75$ years has become a criterion for non-eligibility to IC in recent clinical trials and for novel drug indications. Over the past decade, HMAs have emerged as an alternative to IC in this specific situation [21]. Although these therapeutic strategies have not been formally compared in a prospective randomized trial, most retrospective studies have shown similar median overall survival rates [22-24]. A recent single-center study even reported a survival benefit in favor of HMAs [25]. Thus, there is a dilemma between two options, one associated with greater toxicity but higher response rate and a possibility of long-term survival, and the other, better tolerated, producing fewer responses and no real hope of survival beyond three years. Apart from this, IC requires only short term therapy while HMA treatment should be continued lifelong thus also interfering with long-term quality of life.

In this study, we thought to gather a sufficient number of older European AML patients to compare the effect of IC and HMA treatments by multivariate and propensity score matched analyses. We therefore collected demographic and therapeutic data from 3700 patients aged 70 years or older with the aim of comparing patients routinely selected for IC or HMAs.

\section{SUBJECTS AND METHODS \\ Patients}

Individual patient data were collected from three European AML registries: Toulouse-Bordeaux DATAML, Study Alliance Leukemia (SAL) and Programa Español de Tratamientos en Hematología (PETHEMA). All patients 70 years of age or older with AML newly diagnosed between January 1, 2007, and June 30, 2018 were included. Acute promyelocytic leukemia cases were not included. A minimal data set was collected for each patient, including the variables age, sex, date of diagnosis, AML status (de novo or secondary), white blood cell count, percentage of peripheral and bone marrow blasts, LDH, cytogenetic risk, NPM1, FLT3-ITD, CEBPA, IDH1, IDH2 mutational status, nature of first-line therapy, response to treatment, allogeneic hematopoietic stem cell transplantation in first complete remission, date of relapse and/or death. This study was performed in accordance with the Declaration of Helsinki. All registries were approved by institutional review boards or national authorities, and informed consent was obtained from all patients.

\section{Treatments and endpoints}

First-line treatments included intensive induction chemotherapy combining an anthracycline (idarubicin or daunorubicin) and cytarabine with or without a third drug such as lomustine, a semi-intensive regimen, HMAs (azacitidine or decitabine), low-dose cytarabine (LDAC) or best supportive care. Chemotherapy regimens routinely used by the three study groups have been published elsewhere [9, 26-28]. Semi-intensive regimens in the PETHEMA group included fludarabine, cytarabine, and filgrastim [27]. Allogeneic hematopoietic stem cell transplantation could be offered in selected patients mainly after intensive chemotherapy. Bone marrow assessment in patients treated with intensive chemotherapy was performed after blood recovery or, in case of delayed recovery between days 35 and 45. In the HMA group, bone marrow aspiration was carried out after 3 to 6 cycles. Response to treatment, relapse, relapse-free survival (RFS) and overall survival (OS) were defined according to the European Leukemia Net (ELN) criteria [29].

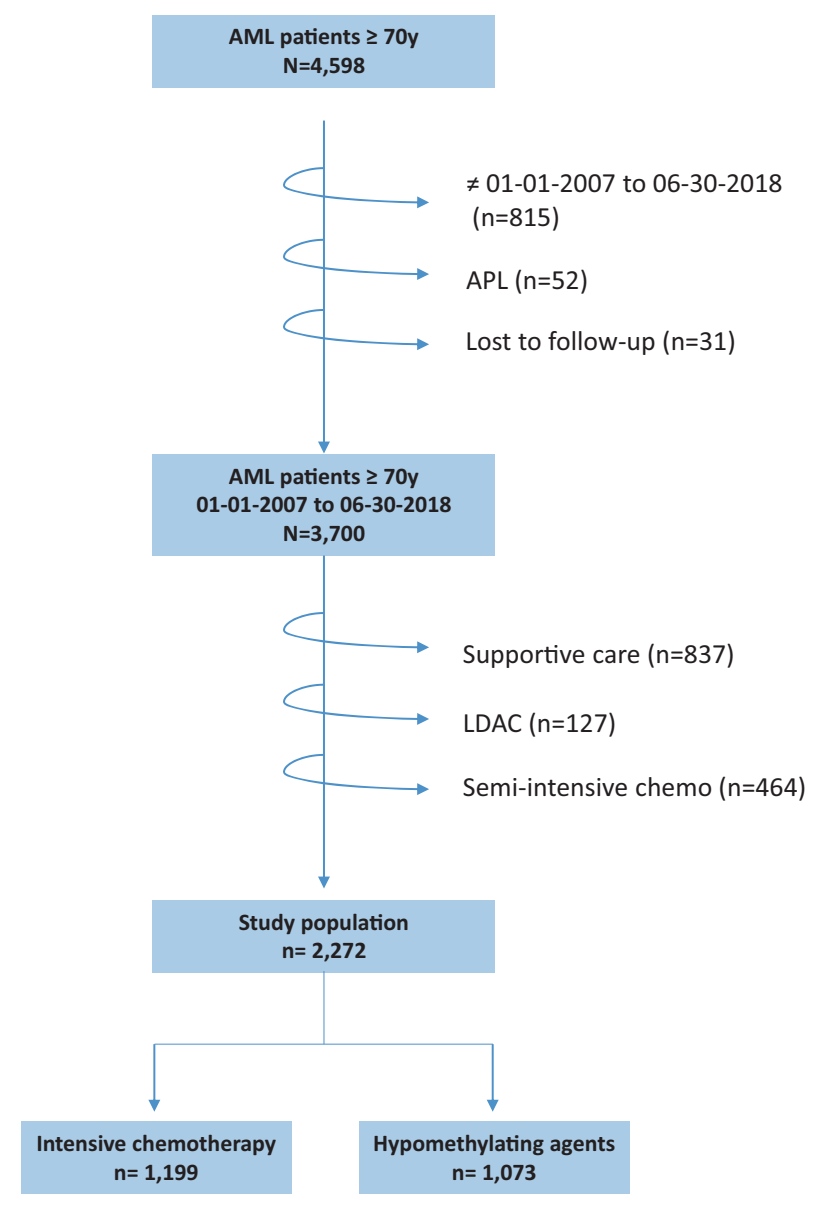

Fig. 1 Study flowchart. APL acute promyelocytic leukemia. LDA low-dose cytarabine.

\section{Statistical analysis}

Statistical analyses were performed using STATA statistical software, version 16.1 (STATA Corp., College Station, TX). We described the patients' characteristics using numbers and frequencies for qualitative data, and medians with inter-quartile ranges (IQR) for quantitative data. Comparisons between the patients' characteristics were assessed using Student's $t$-test (or the Mann-Whitney test when the distribution departed from normality or when homoscedasticity was rejected) for continuous variables, and the X2-test (or Fisher's exact test when there were small expected numbers) for categorical variables. Then, OS and RFS for HMAs vs. IC were described using Kaplan-Meier curves. Because the proportional hazards assumption was not respected for the effect of HMAs vs. IC, we used a Royston and Parmar survival model [30]. Differences in early death and response rate were compared between treatments using a logistic regression model. Multivariate analyses included HMAs vs. IC together with potential confounding factors [age $\geq 75 y$, performance status $>1$, white blood cell count at diagnosis $>30$ giga per liter, cytogenetic risk, secondary vs de novo AML, NPM1 mutation, FLT3-ITD mutation (for RFS) and study period]. Stepwise analysis was then used to assess variables that were significantly and independently associated with the endpoints. Interactions between all potential confounding factors and treatment (HMAs vs. IC) were tested. None were significant, indicating that the effect of HMAs vs. IC is not significantly different according to all confounding factors analyzed (and in particular according to age, performance status, cytogenetic risk or NPM1 mutation). To better appreciate the impact of HMAs vs. IC, we used the propensity score method to more extensively take into account potential baseline differences between HMAs and IC subjects. A multivariate logistic regression model was generated to estimate for each patient a propensity score to receive HMAs vs. IC. Covariates were all variables expected to be associated with HMAs vs. IC in clinical practice (age, performance status, WBC, cytogenetic risk, secondary vs de novo AML, NPM1 and FLT3-ITD mutations, study period and center). The performance of the model was estimated with the $\times 2$-Hosmer-Lemeshow statistic and the C-statistic. 
Table 1. Characteristics of the 2272 AML patients $\geq 70$ years according to treatment.




Based on propensity score, subjects with IC were matched with subjects with HMAs and endpoints were compared between HMAs and IC in the subgroup of propensity score matched subjects. All reported $p$-values were two-sided and the significance threshold was $<0.05$.

\section{RESULTS \\ Study population}

A total of $3700 \mathrm{AML}$ patients $\geq 70$ years of age with sufficient data were identified (Fig. 1). Patients treated with semi-intensive chemotherapy $(n=464)$, LDAC $(n=127)$ or supportive care $(n=$ 837) were not included in the primary analysis (see characteristics and outcome in Supplementary Table 1 and 2). Thus, the study population for the principal objective of this study included 1199 patients treated with IC and 1073 patients treated with HMAs. Their characteristics are presented in Table 1. The median followup was 49.5 months (interquartile range, 29.1-75.0). HMAs were more frequently used in the recent period (2013-2018). This trend was not different between patients aged 70-74 years and patients $\geq 75$ years. In the HMA group, patients were older, had lower WBC count and bone marrow blast percentages, and they more frequently had ECOG performance status $>1$, secondary AML and adverse-risk cytogenetics as compared to the IC group. NPM1 and FLT3-ITD mutations were more frequent in the IC group. The main IC regimens were daunorubicin-cytarabine $(n=432,36.0 \%)$, idarubicin-cytarabine $(n=381,31.8 \%)$ or idarubicin-cytarabinelomustine $(n=214,17.8 \%)$ combinations. Inclusion in a clinical trial involved 141 patients $(11.8 \%)$ treated with IC and 210 $(19.6 \%)$ treated with HMA $(P<0.001)$ without the addition of venetoclax or other antineoplastic agents. Allogeneic hematopoietic stem cell transplantation was performed in 70 patients $(5.8 \%)$ treated with IC and only in seven patients $(0.7 \%)$ treated with HMAs $(P<0.001)$

\section{Response to treatment}

Complete remission or complete remission with incomplete hematologic recovery (CR/CRi) was achieved in 673 (56.1\%) and $211(19.7 \%)$ patients in the IC and HMA groups, respectively $(P<$ $0.0001)$. Multivariate analysis of factors associated with $C R / C R i$ is shown in Table 2. Age $\geq 75$ years, ECOG performance status $>1$, adverse-risk cytogenetics and WBC count $>30$ giga/liter were significantly and independently associated with a lower response rate whereas NPM1 mutation was significantly and independently associated with a higher response rate. After adjusting for these factors, the choice of first-line treatment was also significantly and independently associated with response, meaning that HMA treatment was associated with a lower response rate than IC (odds-ratio (OR), $0.25 ; 95 \% \mathrm{Cl}: 0.20-0.31 ; P<0.001)$.

\section{Early mortality}

Early mortality was evaluated at day 30 and day 60 of treatment in both groups. Day-30 death occurred in 156 (13.0\%) and 93 (8.7\%) patients in the IC and HMA groups, respectively $(P=0.001)$. Multivariate analysis of factors associated with day-30 death showed that ECOG performance status $>1$ and WBC count $>30$ giga/liter were significantly and independently associated with a higher day-30 death rate (Table 2). After adjustment for these factors, the choice of first-line treatment was also significantly and independently associated with day-30 death meaning that HMA treatment was associated with a lower day-30 death rate than IC (OR, 0.61; 95\% Cl: 0.45-0.82; $P=0.001$ ).

Day-60 death occurred in 247 (20.6\%) and 194 (18.1\%) patients in the IC and HMA groups, respectively $(P=0.129)$. Multivariate analysis of factors associated with day- 60 death showed that age $\geq 75$ years, ECOG performance status $>1$, adverse-risk cytogenetics and WBC count $>30$ giga/liter were significantly and independently associated with a higher day-60 death rate (Table 2).
After adjusting for these factors, the choice of first-line treatment was also significantly and independently associated with day- 60 death, meaning that HMA treatment was associated with a lower day-60 death rate than IC (OR, $0.69 ; 95 \% \mathrm{Cl}: 0.54-0.88 ; P=0.003)$.

\section{Overall and relapse-free survival}

The median overall survival was 10.9 (95\% Cl: 9.7-11.6) and 9.2 months (95\% Cl: 8.3-10.2) in the IC and HMA groups, respectively. Overall survival at one, three and five years was 46.0 (95\% Cl: $43.0-48.9)$ vs. $40.6 \%$ (95\% Cl: $37.6-43.7), 20.8$ (95\% Cl: $18.3-23.4)$ vs. $8.3 \%$ (95\% Cl: $6.5-10.4)$ and 12.4 (95\% Cl: $10.2-14.9)$ vs $2.8 \%(95 \% \mathrm{Cl}: 1.7-4.4)$ in the IC and HMA groups, respectively (Fig. 2A). In multivariate analysis, ECOG performance status $>1$, adverse-risk cytogenetics, WBC count $>30$ giga/liter and secondary AML were significantly and independently associated with a poorer overall survival (Table 3). Furthermore, long-term survival ( $>3$ years) was associated with a higher achievement of CR with IC (Supplementary Table 3).

Among the $673 \mathrm{CR} / \mathrm{CRi}$ patients in the IC group, 405 (60.2\%) relapsed, whereas 139 out of $211 \mathrm{CR} / \mathrm{CRi}$ patients in the HMA group (65.9\%) relapsed. Median relapse-free survival was 11.5 months (95\% Cl: $10.5-12.7)$ and 11.0 months $(95 \% \mathrm{Cl}$ : 9.7-12.9) in the IC and HMA groups, respectively (Fig. 2B). It should be noted that relapse-free survival estimates may be biased in favor of IC given the much later time point when remission status was first assessed in patients treated with HMAs (median time between the beginning of treatment and the date of CR: 3.0 months [IQR:1.9-5.6] in the HMA group vs 1.3 months [IQR:1.1-1.8] in the IC group; $\mathrm{p}<0.0001$ ). In multivariate analysis, NPM1 mutation was significantly and independently associated with a better relapse-free survival whereas ECOG performance status $>1$, adverse-risk cytogenetics, FLT3-ITD mutation and secondary AML were associated with a poorer relapse-free survival (Table 2).

Of note, the study period (2007-2012 vs. 2013-2018) was not independently associated with $C R / C R i$, early death rates, relapsefree and overall survival.

\section{Time-dependent treatment effect}

The treatment effect on relapse-free and overall survival was timedependent. To account for the non-proportionality of risks, we used a Royston and Parmar model, which took into account the interactions between time and treatment effect and allowed graphical representation of the adjusted risk of death (or of the adjusted risk of relapse or death for relapse-free survival) at all times during follow-up (Fig. 2C, D). This model showed that patients treated with HMAs had a significantly lower risk of death than patients treated with IC before 1.5 months of follow-up; there was no significant difference between the HMA and IC groups between 1.5 months and 4.0 months, and overall survival was significantly better in the IC group from 4.0 months of follow-up (Fig. 2C). Similarly, for relapse-free survival, patients treated with HMAs had a significantly lower risk of relapse or death than patients treated with IC before 3 months of follow-up; there was no significant difference between 3.0 months and 8.5 months, and relapse-free survival was significantly better in the IC group from 8.5 months of follow-up (Fig. 2D).

Of note, interactions between treatment (HMAs vs. IC) and all confounding factors were tested (for relapse-free survival, overall survival, CR/CRi and early death models). None were significant, indicating that the effect of HMAs vs. IC is not significantly different according to confounding factors (and in particular according to age, performance status, cytogenetic risk or NPM1 mutation). Thus, there is no indication to stratify the analysis in these subgroups. Nevertheless, for information, we showed the results according to age ( $<$ vs $\geq 75 \mathrm{y}$ ) in Supplementary Table 4 and Supplementary Fig. 1. 


\begin{tabular}{|c|c|c|c|c|c|}
\hline & $\begin{array}{l}\text { Number } \\
\text { CR/CRi }\end{array}$ & Events & aOR & $95 \% \mathrm{Cl}$ & $P$ value \\
\hline \multicolumn{6}{|l|}{ Treatment } \\
\hline Intensive chemotherapy & 1199 & 673 & 1 & & \\
\hline Hypomethylating agents & 1073 & 211 & 0.25 & $0.20-0.31$ & $<0.001$ \\
\hline \multicolumn{6}{|l|}{ Age - years } \\
\hline \multicolumn{6}{|l|}{ ECOG performance status } \\
\hline $0-1$ & 1497 & 661 & 1 & & \\
\hline $2-4$ & 587 & 159 & 0.52 & $0.41-0.65$ & $<0.001$ \\
\hline \multicolumn{6}{|l|}{ AML status } \\
\hline Intermediate & 1313 & 610 & 0.54 & $0.30-0.98$ & 0.043 \\
\hline Adverse & 557 & 148 & 0.32 & $0.17-0.59$ & $<0.001$ \\
\hline \multicolumn{6}{|c|}{ White blood cell count - giga per liter } \\
\hline$\leq 30$ & 1707 & 644 & 1 & $0.52-0.84$ & \\
\hline$>30$ & 518 & 222 & 0.66 & & 0.001 \\
\hline \multicolumn{6}{|l|}{ NPM1 mutations } \\
\hline No & 943 & 382 & 1 & $1.36-2.36$ & \\
\hline \multirow[t]{2}{*}{ Yes } & 396 & 230 & 1.79 & & $<0.001$ \\
\hline & \multicolumn{5}{|c|}{ Day-30 death } \\
\hline Favorable & 56 & 3 & 1 & & \\
\hline Intermediate & 1313 & 123 & 1.96 & $0.60-6.46$ & 0.268 \\
\hline Adverse & 557 & 68 & 3.26 & $0.97-10.93$ & 0.055 \\
\hline \multicolumn{6}{|c|}{ White blood cell count - giga per liter } \\
\hline$\leq 30$ & 1707 & 151 & 1 & & \\
\hline \multirow[t]{2}{*}{$>30$} & 518 & 95 & 1.99 & $1.47-2.69$ & $<0.001$ \\
\hline & Day-60 de & & & & \\
\hline \multicolumn{6}{|l|}{ Treatment } \\
\hline Intensive chemotherapy & 1199 & 247 & 1 & & \\
\hline Hypomethylating agents & 1073 & 194 & 0.69 & $0.54-0.88$ & 0.003 \\
\hline \multicolumn{6}{|l|}{ Age - years } \\
\hline$<75$ & 1071 & 187 & 1 & & \\
\hline$\geq 75$ & 1201 & 254 & 1.29 & $1.02-1.61$ & 0.030 \\
\hline \multicolumn{6}{|l|}{ ECOG performance status } \\
\hline $0-1$ & 1497 & 222 & 1 & & \\
\hline $2-4$ & 587 & 187 & 2.50 & $1.99-3.16$ & $<0.001$ \\
\hline \multicolumn{6}{|l|}{ Cytogenetic risk } \\
\hline Favorable & 56 & 5 & 1 & & \\
\hline Intermediate & 1313 & 207 & 1.97 & $0.77-5.06$ & 0.160 \\
\hline
\end{tabular}


Table 2 continued

\section{Day-60 death}

White blood cell count - giga per liter

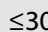

$>30$
1707

518
300

136
1

1.56

$1.21-2.02$ 0.001

$a O R$ adjusted odds ratio, $C l$ confidence interval, $C R$ complete remission, $C R i$ complete remission with incomplete hematologic recovery.

Interaction between treatment (hypomethylating agents vs. intensive chemotherapy) and age (<vs $\geq 75 \mathrm{y})$, performance status ( $\leq$ vs $>1$ ), cytogenetic risk (favorable vs. intermediate vs. adverse) or NPM1 mutation (yes vs. no) was not significant, showing that the effect of hypomethylating agents vs. intensive chemotherapy was not significantly different according to age, performance status, cytogenetic risk and NPM1 mutation. Thus, there is no indication to stratify the analysis of age, performance status, cytogenetic risk and NPM1 mutation (the OR for hypomethylating agents vs. intensive chemotherapy shown in Table 2 was the same according to age (< vs $\geq 75 \mathrm{y}$ ), performance status ( $\leq$ vs $>1$ ), cytogenetic risk (favorable vs. intermediate vs. adverse) or NPM1 mutation (yes vs. no)).

\section{Propensity score matching}

To better estimate the impact of treatment on endpoints, we used the propensity score method to more extensively take into account potential baseline differences between HMA and IC subjects. A multivariate logistic regression model was generated to estimate for each patient a propensity score to receive HMAs or IC. The performance of the model was estimated with the $x^{2}$ Hosmer-Lemeshow statistic $(P$ value $=0.169)$ and the C-statistic (0.82, 95\% Cl: $0.81-0.84)$. The mean propensity score was 0.320 $( \pm 0.232)$ in IC $(N=1199)$ and $0.642( \pm 0.234)$ in HMA $(N=1073)$. Based on propensity score, 532 subjects with IC were matched with 532 subjects with HMAs (630 with a precision of 0.0001 , 18 with a precision of $0.001,148$ with a precision of 0.01 and the last with a precision of 0.1$)$. The mean propensity score was the same in IC and HMA $(0.491 \pm 0.219)$ in the matched sample. The results of HMAs vs. IC comparisons on response, early mortality, disease-free survival and overall survival in this subgroup of propensity score-matched patients were similar to those of the multivariate analysis (Supplementary Table 5 and Fig. 3). Median relapse-free survival was 11.9 (95\% Cl: $10.3-14.5)$ and $10.0(95 \% \mathrm{Cl}$ : 8.4-12.9) months in the IC and HMA groups, respectively. Median overall survival was 10.5 (95\% Cl: 8.8-12.2) and $9.6(95 \% \mathrm{Cl}$ : 8.5-11.0) months in the IC and HMA groups, respectively. Again, the treatment effect on relapse-free and overall survival was time-dependent. The Royston and Parmar model assessing the evolution of hazard ratios according to time is presented in Fig. 3.

\section{DISCUSSION}

This study is the largest multicenter comparison of the two most commonly used front-line therapies in AML patients $\geq 70$ years. With a fairly long median follow-up in this patient population, we showed that IC remains the treatment strategy that offers better chances for prolonged survival compared with HMAs. No significant interaction was found between treatments and independent variables indicating that the effect of treatment was not significantly different across the different subsets of patients, including those aged $\geq 75$ years, with a poor performance status or even with an adverse cytogenetic risk.

After a decade of experience with HMAs in older AML patients, efforts to make comparisons with IC have been challenging and controversial, both prospectively and retrospectively [21]. In the prospective AZA-AML-001 randomized trial, IC was part of a conventional care regimen together with LDAC and best supportive care making a head-to-head comparison virtually impossible. Indeed, in this trial comparing azacitidine vs. a conventional care regimen, only 44 patients were assigned to IC [31]. Retrospective studies, which were most often monocentric and underpowered, yielded contradictory results [22-25]. A recent single-center study from the Moffitt Cancer Center conducted in
AML patients $\geq 70$ years showed opposite results to our analysis [25]. In this study, HMA treatment was associated with a significant better overall survival compared to IC. However, there are several important differences between both studies regarding the baseline characteristics of patients that may explain these discrepancies. These differences concerned the rates of secondary AML ( $56.9 \%$ vs. $32.4 \%$ in our study) and performance status $>1$ (19\% vs. $28 \%)$, white blood cell count (median, 3.3 vs. 5.2 giga per liter) and NPM1 mutations $(12.2 \%$ vs. $29.6 \%$ ). Thus, the patient population of the Moffit Cancer Center presented more often with AML with myelodysplastic syndrome-like features, suggesting a center-related recruitment bias compared to our multicentric cohort. Study periods were also different with a more recent patient population in our study. Moreover, the median follow up in our study was much longer (49.5 vs. 20.5 months), which may be relevant regarding long-term survival results.

There is no doubt that IC remains more toxic than HMAs in older AML patients as reflected by the higher early death rate in our study. However, the rate of early death following induction chemotherapy has decreased over time, and new intensive chemotherapy formulations such as CPX-351 may further limit this risk and allow more patients to reach response and go to consolidation and/or maintenance therapy with novel agents such as CC-486 [32-34]. The age limit for allogeneic hematopoietic stem cell transplantation is also rising constantly and patients $\geq 70$ years achieving complete remission may now become candidates for transplantation more and more frequently [35].

Obviously, these results should be discussed in light of recent advances in the treatment of AML patients judged unfit for IC. The addition of venetoclax to low-intensity therapies including HMA or LDAC has demonstrated significant efficacy by improving response rates and overall survival compared to HMA or LDAC single agents $[10,11]$. In the VIALE-A study comparing venetoclax/ azacitidine vs. placebo/azacitidine, the venetoclax experimental arm (median age, 76 years, de novo AML 75\%, intermediate-risk cytogenetics $64 \%$ ) induced a $\mathrm{CR} / \mathrm{CRi}$ rate of $66.4 \%$, which represents a real breakthrough in the field of low-intensity therapies, reaching the level of IC results. It is noteworthy that age $\geq 75$ years or a performance status of 2 were isolated criteria sufficient to be judged ineligible for IC, which is debatable. Median overall survival was 14.7 months and two-year survival $~ 35 \%$. Since previous studies have shown similar median overall survival between HMAs and $\mathrm{IC}$ as discussed above, it is tempting to speculate that venetoclax plus low-intensity therapies will therefore be superior to IC (and likely less toxic) in older AML patients. However, the median follow-up of VIALE-A (20.5 months) is not yet long enough to determine the long-term survival rate with this novel therapeutic approach. Moreover, the first real-world experience with venetoclax/azacitidine suggested an inferior outcome to the clinical trial results [36]. Therefore, prospective 
A

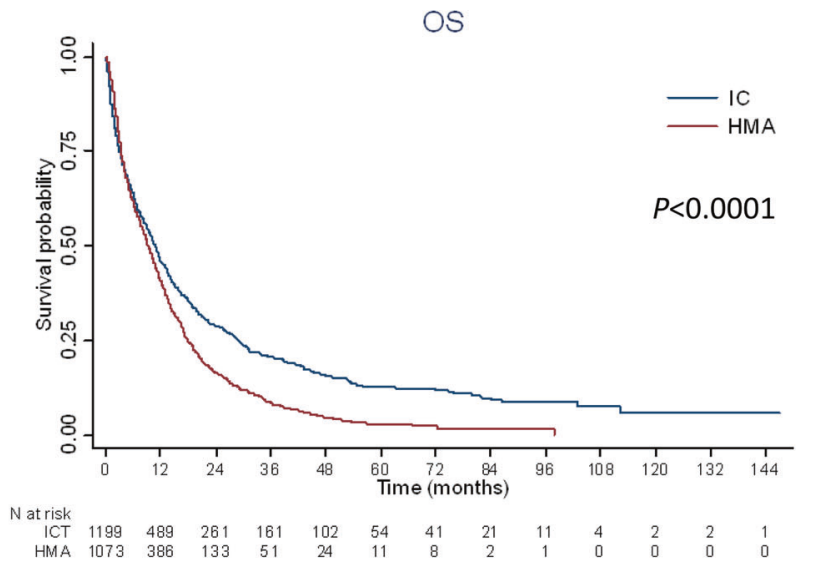

C

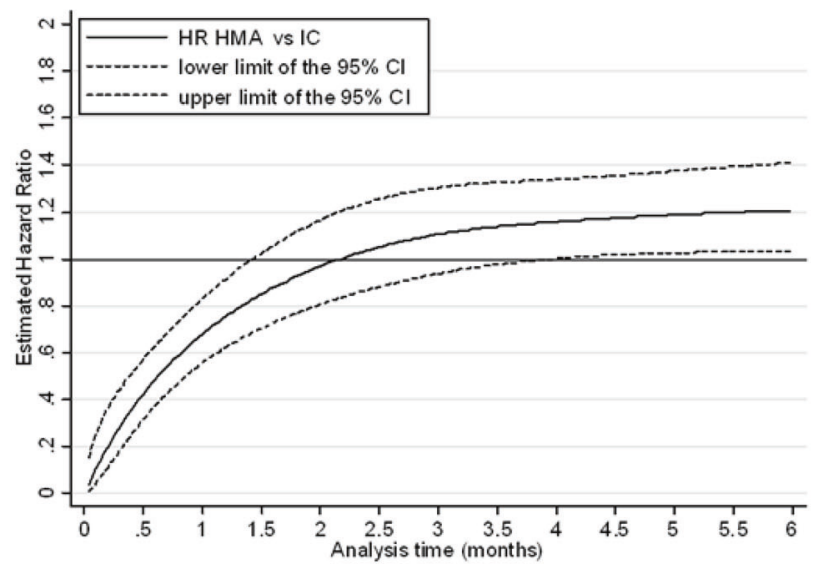

B

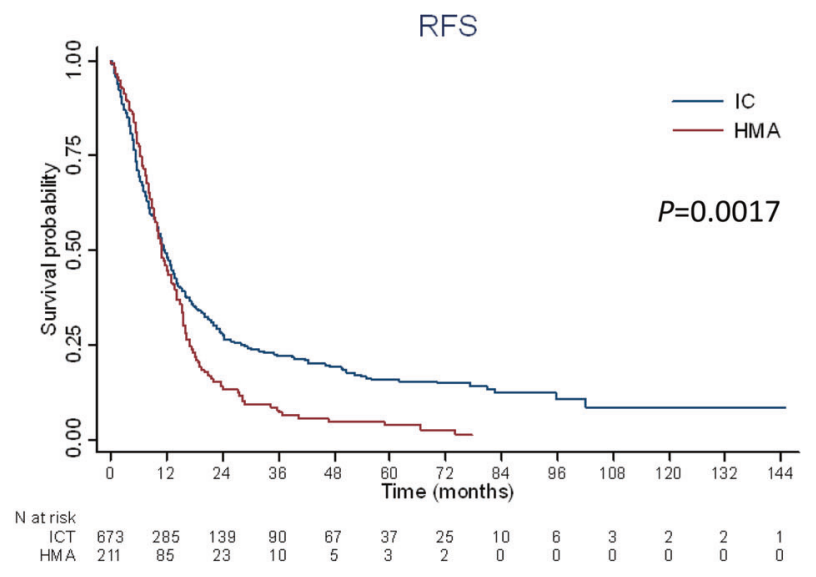

D

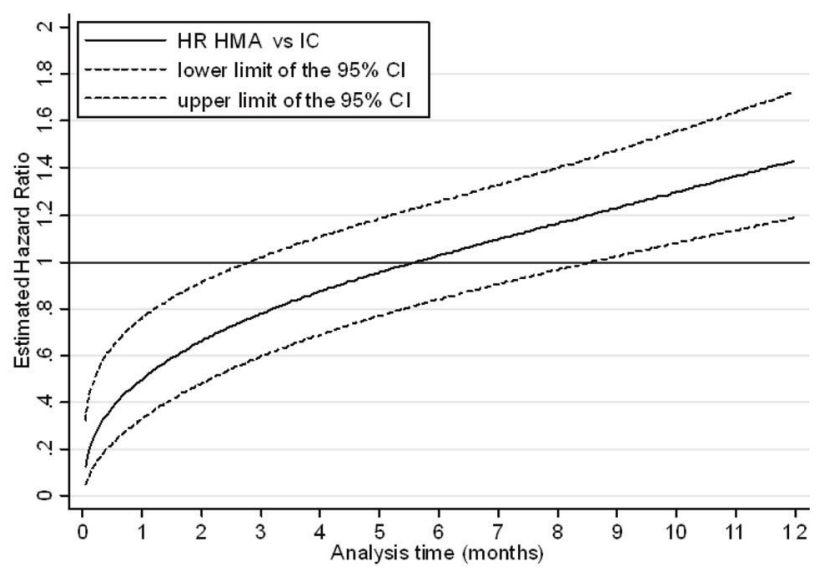

Fig. 2 Survival according to intensive chemotherapy or HMA treatment. A Kaplan-Meier curve of overall survival according to treatment (median OS: 10.9 months, 95\% Cl: 9.7-11.6 with IC and 9.2 months, 95\% Cl: 8.3-10.2 with HMAs). B Kaplan-Meier curve of relapse free survival according to treatment (median RFS: 11.5 months, 95\% Cl: 10.5-12.7 with IC and 11.0 months, 95\% Cl: 9.7-12.9 with HMAs). C Royston and Parmar adjusted* hazard ratio for overall survival in HMA vs. IC for each month from diagnosis. Before 1.5 months of follow-up, patients treated with HMAs had a significantly lower risk of death compared to IC patients. Between 1.5 months and 4.0 months of follow-up, there was no significant difference in survival between HMAs and IC patients. From 4.0 months of follow-up, patients treated with HMAs had a significantly higher risk of death compared to IC patients. Interaction between treatment (HMAs vs. IC) and age $(<$ vs $\geq 75 \mathrm{y})$, performance status ( $\leq$ vs $>1$ ), cytogenetic risk (favorable vs. intermediate vs. adverse) or NPM1 mutation (yes vs. no) was not significant, showing that the effect of HMAs vs. IC was not significantly different according to age, performance status, cytogenetic risk and NPM1 mutation. Thus, there is no indication to stratify the analysis on age, performance status, cytogenetic risk and NPM1 mutation (Figure C was the same according to age (< vs. $\geq 75$ y), performance status ( $\leq$ vs. $>1$ ), cytogenetic risk (favorable vs. intermediate vs. adverse) or NPM1 mutation (yes vs. no)). ${ }^{*}$ Adjusted for age $\geq 75 \mathrm{y}$, performance status $>1$, white blood cell count at diagnosis $>30$ giga per liter, cytogenetic risk, secondary vs de novo AML and NPM1 mutation. D Royston and Parmar adjusted* hazard ratio for relapse-free survival in HMAs vs. IC for each month from CR/CRi. Before 3 months of follow-up, patients treated with HMAs had a significantly lower risk of relapse or death compared to IC patients. Between 3 months and 8.5 months from CR/CRi, there was no significant difference between HMAs and IC patients. Beyond 8.5 months from CR/CRi, patients treated with HMA had a significantly higher risk of relapse or death compared to IC patients. Interaction between treatment (HMAs vs. IC) and age (< vs. $\geq 75$ y), performance status ( $\leq$ vs. $>1$ ), cytogenetic risk (favorable/intermediate vs. adverse) or NPM1 mutation (yes vs. no) was not significant, showing that the effect of HMAs vs. IC was not significantly different according to age, performance status, cytogenetic risk and NPM1 mutation. Thus, there is no indication to stratify the analysis on age, performance status, cytogenetic risk and NPM1 mutation (Figure D was the same according to age $(<\mathrm{vs}$. $\geq 75 \mathrm{y}$ ), performance status ( $\leq$ vs. $>1)$, cytogenetic risk (favorable/intermediate vs. adverse) or NPM1 mutation (yes vs. no)). *Adjusted for performance status $>1$, white blood cell count at diagnosis $>30$ giga per liter, cytogenetic risk, secondary vs. de novo AML, NPM1 and FLT3-ITD mutations.

randomized trials will be necessary to answer this important clinical issue.

The main weakness of our study is its retrospective nature and the lack of predefined criteria to justify the therapeutic options proposed in real life. Molecular documentation remains insufficient, even if we could show the prognostic impact of NPM1 and FLT3-ITD mutations. However, the multicenter and multinational aspect, as well as the very high number of patients allowed robust multivariate and matching analyses that shed light on the respective place of IC and HMAs in patients 70 years of age or older. 
Table 3. Multivariate analysis for overall and relapse free survival.

\begin{tabular}{|c|c|c|c|c|c|}
\hline & $\begin{array}{l}\text { Number } \\
\text { Overall survival }\end{array}$ & Events & aHR & $95 \% \mathrm{Cl}$ & $P$ value \\
\hline Intensive chemotherapy & 1199 & 928 & & & \\
\hline \multicolumn{6}{|l|}{ Age - years } \\
\hline$<75$ & 1071 & 839 & 1 & & \\
\hline $0-1$ & 1497 & 1189 & 1 & & \\
\hline $2-4$ & 587 & 507 & 1.56 & $1.41-1.74$ & $<0.001$ \\
\hline \multicolumn{6}{|l|}{ AML status } \\
\hline De novo & 1420 & 1108 & 1 & & \\
\hline Secondary & 680 & 597 & 1.21 & $1.09-1.34$ & $<0.001$ \\
\hline Adverse & 557 & 495 & 1.73 & $1.25-2.39$ & 0.001 \\
\hline \multicolumn{6}{|c|}{ White blood cell count - giga per liter } \\
\hline$\leq 30$ & 1707 & 1396 & 1 & & \\
\hline$>30$ & 518 & 413 & 1.40 & $1.25-1.57$ & $<0.001$ \\
\hline \multicolumn{6}{|l|}{ NPM1 mutations } \\
\hline No & 1383 & 1146 & 1 & & \\
\hline Yes & 546 & 409 & 0.82 & $0.71-0.95$ & 0.010 \\
\hline
\end{tabular}

\section{Treatment}

Intensive chemotherapy
Hypomethylating agents
COG performance status

Relapse-free survival

$0-1$

673

179 See R\&P (Fig. 2D)

$661 \quad 500 \quad 1$

AML status

De novo

1

Cytogenetic risk

174

Favorable/ Intermediate

1 1.58

White blood cell count - giga per liter

$$
\leq 30
$$

$$
644
$$

500

1

167

1.19

0.98-1.45

\section{FLT3-ITD mutations}

No

511

386

1

114

90

1.33

$1.03-1.73$

0.029

NPM1 mutations

No

382

303

1

230

160

0.71

$0.57-0.87$

0.001

aHR, adjusted hazard ratio; $\mathrm{Cl}$, confidence interval. ${ }^{a}$ R\&P: see Royston \& Parmar.

Although IC results remains largely unsatisfactory in AML of the elderly, it is associated with short-term toxicities but long-term survival in a sizeable number of patients. The main result of our study suggests that the evaluation of new alternative treatments should integrate long term survival as a relevant clinical endpoint in order to have a clear vision of the benefits of prolonged lowintensity treatments compared to short IC, even in elderly subjects. 
A

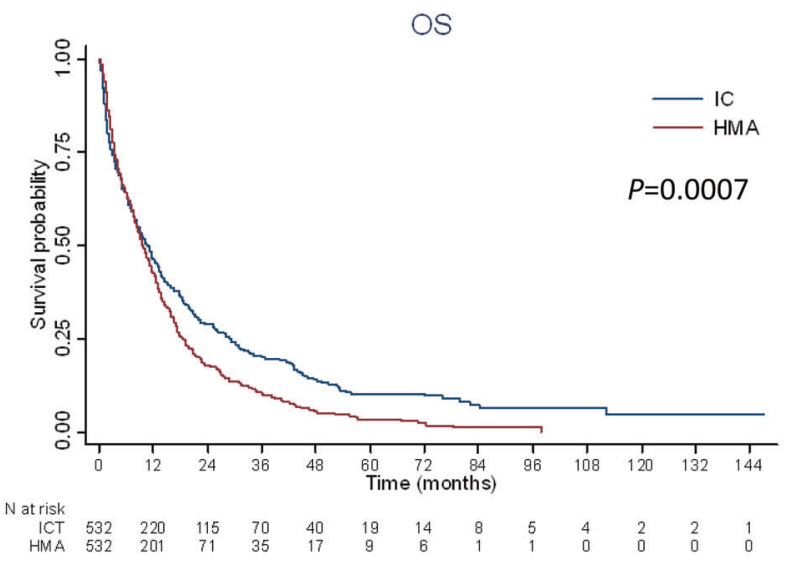

C

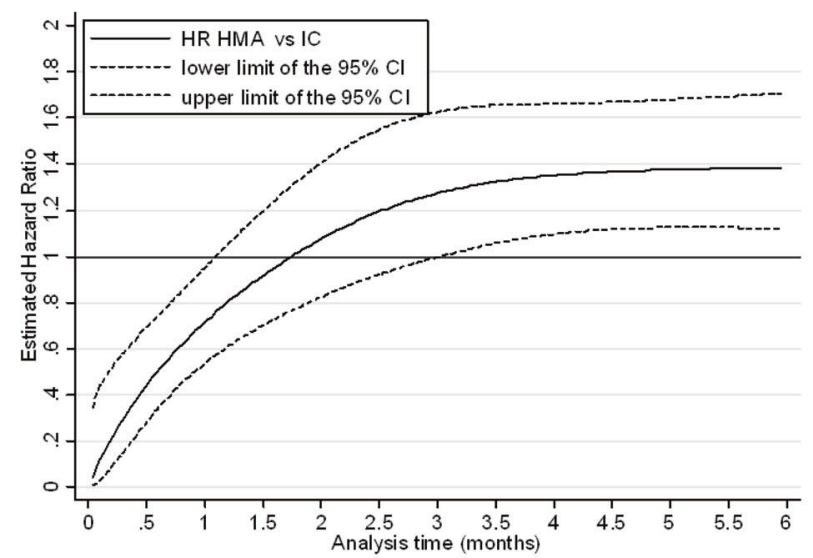

B

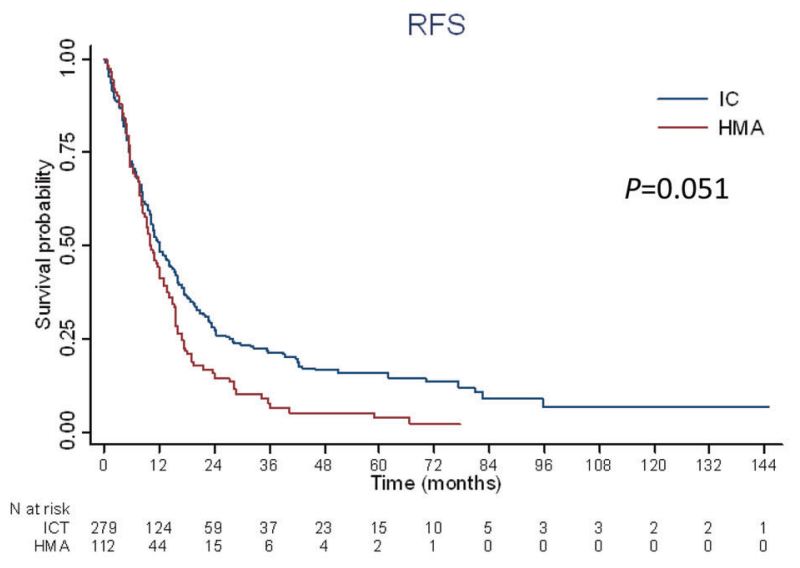

D

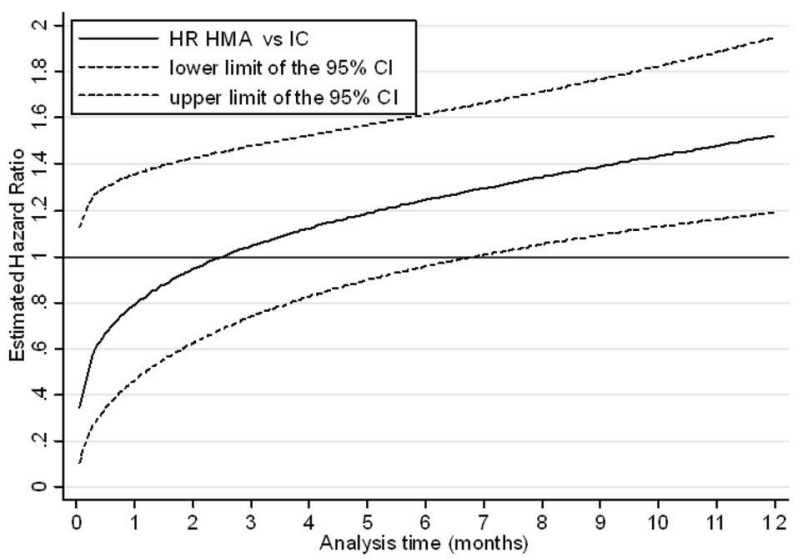

Fig. 3 Survival according to intensive chemotherapy or HMA treatment in the pairwise population matched by the propensity score. A Kaplan-Meier curve of overall survival according to treatment in 532 IC patients matched with 532 HMA patients (median OS: 10.5 months, 95\% Cl: 8.8-12.2, with IC and 9.6 months, 95\% Cl: 8.5-11.0, with HMAs). B Kaplan-Meier curve of relapse free survival according to treatment (median RFS: 11.9 months, 95\% Cl: 10.3-14.5, with IC and 10.0 months, 95\% Cl: 8.4-12.9, with HMAs). C Royston and Parmar hazard ratio for overall survival in HMAs vs. IC for each month from diagnosis. Before one month of follow-up, patients treated with HMAs had a significantly lower risk of death compared to IC patients. Between 1 month and 3.0 months of follow-up, there was no significant difference in survival between HMA and IC patients. From 3.0 months of follow-up, patients treated with HMAs had a significantly higher risk of death compared to IC patients. D Royston and Parmar hazard ratio for relapse-free survival in HMAs vs. IC for each month from CR/CRi. Before 7 months of followup from CR/CRi, there was no significant difference in relapse or death between HMA and IC patients. From seven months of follow-up after $\mathrm{CR} / \mathrm{CRi}$, patients treated with HMAs had a significantly higher risk of relapse or death compared to IC patients.

\section{REFERENCES}

1. Shallis RM, Wang R, Davidoff A, Ma X, Zeidan AM. Epidemiology of acute myeloid leukemia: Recent progress and enduring challenges. Blood Rev. 2019;36:70-87.

2. Sant M, Minicozzi P, Mounier M, Anderson LA, Brenner H, Holleczek B, et al. Survival for haematological malignancies in Europe between 1997 and 2008 by region and age: results of EUROCARE-5, a population-based study. Lancet Oncol 2014; 15:931-42.

3. Podoltsev NA, Stahl M, Zeidan AM, Gore SD. Selecting initial treatment of acute myeloid leukaemia in older adults. Blood Rev. 2017;31:43-62.

4. Sorror ML, Storer BE, Fathi AT, Gerds AT, Medeiros BC, Shami P, et al. Development and validation of a novel acute myeloid leukemia-composite model to estimate risks of mortality. JAMA Oncol. 2017;3:1675-82.

5. Appelbaum FR, Gundacker H, Head DR, Slovak ML, Willman CL, Godwin JE, et al. Age and acute myeloid leukemia. Blood 2006;107:3481-5.

6. Bullinger L, Dohner K, Dohner H. Genomics of acute myeloid leukemia diagnosis and pathways. J Clin Oncol. 2017;35:934-46.

7. Tsai CH, Hou HA, Tang JL, Liu CY, Lin CC, Chou WC, et al. Genetic alterations and their clinical implications in older patients with acute myeloid leukemia. Leukemia 2016;30:1485-92.
8. Ellison LF. Increasing survival from leukemia among adolescents and adults in Canada: a closer look. Health Rep. 2016;27:19-26.

9. Bertoli S, Tavitian S, Huynh A, Borel C, Guenounou S, Luquet I, et al. Improved outcome for AML patients over the years 2000-2014. Blood. Cancer J. 2017;7:635.

10. DiNardo CD, Jonas BA, Pullarkat V, Thirman MJ, Garcia JS, Wei AH, et al. Azacitidine and venetoclax in previously untreated acute myeloid leukemia. N. Engl J Med. 2020;383:617-29.

11. Wei $A H$, Montesinos $P$, Ivanov V, DiNardo $C D$, Novak J, Laribi $K$, et al. Venetoclax plus LDAC for newly diagnosed AML ineligible for intensive chemotherapy: a phase 3 randomized placebo-controlled trial. Blood 2020;135:2137-45.

12. Yang J, Schiffer CA. The artful management of older patients with acute myeloid leukemia. Expert Rev Hematol. 2016;9:425-32.

13. Giles FJ, Borthakur G, Ravandi F, Faderl S, Verstovsek S, Thomas D, et al. The haematopoietic cell transplantation comorbidity index score is predictive of early death and survival in patients over 60 years of age receiving induction therapy for acute myeloid leukaemia. Br J Haematol. 2007;136:624-7.

14. Malfuson JV, Etienne A, Turlure $P$, de Revel $T$, Thomas $X$, Contentin $N$, et al. Risk factors and decision criteria for intensive chemotherapy in older patients with acute myeloid leukemia. Haematologica 2008;93:1806-13. 
15. Wheatley K, Brookes CL, Howman AJ, Goldstone AH, Milligan DW, Prentice AG, et al. Prognostic factor analysis of the survival of elderly patients with $A M L$ in the MRC AML11 and LRF AML14 trials. Br J Haematol. 2009;145:598-605.

16. Kantarjian H, Ravandi F, O'Brien S, Cortes J, Faderl S, Garcia-Manero G, et al. Intensive chemotherapy does not benefit most older patients (age 70 years or older) with acute myeloid leukemia. Blood 2010;116:4422-9.

17. Krug U, Rollig C, Koschmieder A, Heinecke A, Sauerland MC, Schaich M, et al. Complete remission and early death after intensive chemotherapy in patients aged 60 years or older with acute myeloid leukaemia: a web-based application for prediction of outcomes. Lancet 2010;376:2000-8.

18. Röllig C, Thiede C, Gramatzki M, Aulitzky W, Bodenstein H, Bornhäuser M, et al. A novel prognostic model in elderly patients with acute myeloid leukemia: results of 909 patients entered into the prospective AML96 trial. Blood 2010;116:971-8.

19. Ferrara F, Barosi G, Venditti A, Angelucci E, Gobbi M, Pane F, et al. Consensusbased definition of unfitness to intensive and non-intensive chemotherapy in acute myeloid leukemia: a project of SIE, SIES and GITMO group on a new tool for therapy decision making. Leukemia 2013;27:997-9.

20. Bories P, Lamy S, Simand C, Bertoli S, Delpierre C, Malak S, et al. Physician uncertainty aversion impacts medical decision making for older patients with acute myeloid leukemia: results of a national survey. Haematologica 2018;103:2040-8.

21. Dombret $H$, Itzykson R. How and when to decide between epigenetic therapy and chemotherapy in patients with AML. Hematol Am Soc Hematol Educ Program. 2017;2017:45-53.

22. Dumas PY, Bertoli S, Berard E, Mediavilla C, Yon E, Tavitian S, et al. Azacitidine or intensive chemotherapy for older patients with secondary or therapy-related acute myeloid leukemia. Oncotarget 2017;8:79126-36.

23. Bories P, Bertoli S, Berard E, Laurent J, Duchayne E, Sarry A, et al. Intensive chemotherapy, azacitidine, or supportive care in older acute myeloid leukemia patients: an analysis from a regional healthcare network. Am J Hematol. 2014;89: E244-52.

24. Quintás-Cardama A, Ravandi F, Liu-Dumlao T, Brandt M, Faderl S, Pierce S, et al. Epigenetic therapy is associated with similar survival compared with intensive chemotherapy in older patients with newly diagnosed acute myeloid leukemia. Blood 2012;120:4840-5.

25. Talati C, Dhulipala VC, Extermann MT, Ali NA, Kim J, Komrokji R, et al. Comparisons of commonly used front-line regimens on survival outcomes in patients aged 70 years and older with acute myeloid leukemia. Haematologica 2020;105:398-406.

26. Röllig C, Kramer M, Schliemann C, Mikesch JH, Steffen B, Krämer A, et al. Does time from diagnosis to treatment affect the prognosis of patients with newly diagnosed acute myeloid leukemia? Blood 2020;136:823-30.

27. Vives S, Martínez-Cuadrón D, Bergua Burgues J, Algarra L, Tormo M, MartínezSánchez MP, et al. A phase 3 trial of azacitidine versus a semi-intensive fludarabine and cytarabine schedule in older patients with untreated acute myeloid leukemia. Cancer 2021;127:2003-14.

28. Martínez-Cuadrón D, Serrano J, Gil C, Tormo M, Martínez-Sánchez P, Pérez-Simón $J A$, et al. Evolving treatment patterns and outcomes in older patients ( $\geq 60$ years) with AML: changing everything to change nothing? Leukemia 2021;35:1571-85.

29. Döhner H, Estey E, Grimwade D, Amadori S, Appelbaum FR, Büchner T, et al. Diagnosis and management of AML in adults: 2017 ELN recommendations from an international expert panel. Blood 2017;129:424-47.

30. Royston P, Lambert PC. Flexible parametric survival analysis using stata: beyond the Cox model: StataCorp LP; 2011.

31. Dombret H, Seymour JF, Butrym A, Wierzbowska A, Selleslag D, Jang JH, et al. International phase 3 study of azacitidine vs conventional care regimens in older patients with newly diagnosed AML with >30\% blasts. Blood 2015;126:291-9.

32. Othus M, Kantarjian H, Petersdorf S, Ravandi F, Godwin J, Cortes J, et al. Declining rates of treatment-related mortality in patients with newly diagnosed AML given 'intense' induction regimens: a report from SWOG and MD Anderson. Leukemia 2014;28:289-92.

33. Lancet JE, Uy GL, Cortes JE, Newell LF, Lin TL, Ritchie EK, et al. CPX-351 (cytarabine and daunorubicin) liposome for injection versus conventional cytarabine plus daunorubicin in older patients with newly diagnosed secondary acute myeloid leukemia. J Clin Oncol. 2018;36:2684-92.

34. Wei AH, Döhner $\mathrm{H}$, Pocock $\mathrm{C}$, Montesinos $\mathrm{P}$, Afanasyev B, Dombret $\mathrm{H}$, et al. Oral azacitidine maintenance therapy for acute myeloid leukemia in first remission. $\mathrm{N}$ Engl J Med. 2020;383:2526-37.

35. Magliano G, Bacigalupo A. Allogeneic hematopoietic stem cell transplantation for acute myeloid leukemia of the elderly: review of literature and new perspectives. Mediterr J Hematol Infect Dis. 2020;12:e2020081.
36. Winters AC, Gutman JA, Purev E, Nakic M, Tobin J, Chase S, et al. Real-world experience of venetoclax with azacitidine for untreated patients with acute myeloid leukemia. Blood Adv. 2019;3:2911-9.

\section{ACKNOWLEDGEMENTS}

We would like to thank the data management unit of Toulouse University Hospital. We thank all the members of the G.A.E.L (Gaël Adolescent Espoir Leucémie) association, the Toulouse Cancer Santé Foundation and the FONROGA Foundation. The authors thank the central study office and all member sites of the Study Alliance Leukemia (SAL) study group.

\section{AUTHOR CONTRIBUTIONS}

CRo, SB, PYD, ST, MK, HS, MB, UP, CMT, CDB, DMC, JS, PMS, ERA, CG, JB, TB, AFB, ED, $A B, A P$ and $P M$, collected data, treated patients or performed molecular analyses. $E B$ performed statistical analysis and wrote the paper. $C R$ collected data, treated patients, supervised analysis and wrote the paper. The manuscript was prepared by all authors with the assistance of a medical writer funded by the $\mathrm{CHU}$ Toulouse. All authors reviewed, provided comments and approved the manuscript.

\section{COMPETING INTERESTS}

Christian Récher: Research grants from AbbVie, Amgen, Novartis, Celgene, Jazz Pharmaceuticals, Agios, Chugai, MaaT Pharma, Astellas, Roche and Daiichi-Sankyo; an advisory role for AbbVie, Janssen, Jazz Pharmaceuticals, Novartis, Celgene, Otsuka, Astellas, Daiichi-Sankyo, Macrogenics, Roche, Takeda and Pfizer. Christoph Röllig: Research grants from AbbVie, Bayer, Celgene, Janssen, Novartis, Pfizer; advisory role for AbbVie, Amgen, BMS/Celgene, Daiichy Sankyo, Janssen, Jazz, Novartis, Pfizer, Roche, Takeda. Pau Montesinos: Research grants from AbbVie, Novartis, BMSCelgene, Jazz Pharmaceuticals, Astellas, and Daiichi-Sankyo; an advisory role for AbbVie, Janssen, Jazz Pharmaceuticals, Novartis, Celgene, Astellas, Daiichi-Sankyo, Roche, Takeda and Pfizer. Arnaud Pigneux: Grant/Research Support: Astellas, Roche; Speaker's Bureau: Astellas, AbbVie, Gilead, Pfizer, Roche, Sanofi; Consultant: Jazz, AbbVie, Agios, BMS, Gilead, Novartis, Pfizer, Roche, Takeda. Michael Kramer: consultant for GEMoaB and Cellex Patient Treatment. Audrey Bidet: advisory boards for Daiichi-Sankyo and Novartis. Pierre-Yves Dumas: Daiichi-Sankyo, Jazz Pharmaceutical, Astellas, AbbVie, Celgene, Janssen. Sarah Bertoli: advisory role for Jazz Pharmaceuticals, Daiichi-Sankyo, Sanofi, Astellas and BMS.

\section{ADDITIONAL INFORMATION}

Supplementary information The online version contains supplementary material available at https://doi.org/10.1038/s41375-021-01425-9.

Correspondence and requests for materials should be addressed to Christian Récher.

Reprints and permission information is available at http://www.nature.com/ reprints

Publisher's note Springer Nature remains neutral with regard to jurisdictional claims in published maps and institutional affiliations.

Open Access This article is licensed under a Creative Commons Attribution 4.0 International License, which permits use, sharing, adaptation, distribution and reproduction in any medium or format, as long as you give appropriate credit to the original author(s) and the source, provide a link to the Creative Commons license, and indicate if changes were made. The images or other third party material in this article are included in the article's Creative Commons license, unless indicated otherwise in a credit line to the material. If material is not included in the article's Creative Commons license and your intended use is not permitted by statutory regulation or exceeds the permitted use, you will need to obtain permission directly from the copyright holder. To view a copy of this license, visit http://creativecommons. org/licenses/by/4.0/.

(c) The Author(s) 2021 